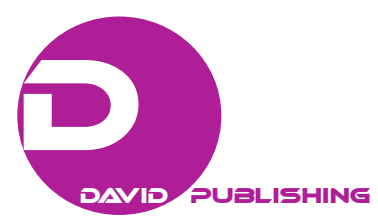

\title{
Discriminant Analysis of the Linear Separable Data - Japanese 44 Cars -
}

\author{
Shuichi Shinmura \\ Faculty of Economics, Seikei University, Japan
}

There are four serious problems in the discriminant analysis. We developed an optimal linear discriminant function (optimal LDF) based on the minimum number of misclassification (minimum NM) using integer programming (IP). We call this LDF as Revised IP-OLDF. Only this LDF can discriminate the cases on the discriminant hyperplane (Problem1). This LDF and a hard-margin SVM (H-SVM) can discriminate the lineary separable data (LSD) exactly. Another LDFs may not discriminate the LSD theoretically (Problem2). When Revised IP-OLDF discriminate the Swiss banknote data with six variables, we find MNM of two-variables model such as (X4, X6) is zero. Because MNMk decreases monotounusly (MNMk >= MNM(k+1)), sixteen MNMs including (X4, X6) are zero. Until now, because there is no research of the LSD, we surveyed another three linear separable data sets such as: 18 exam scores data sets, the Japanese 44 cars data and six microarray datasets. When we discriminate the exam scores with MNM=0, we find the generalized inverse matrix technique causes the serious Problem 3 and confirmed this fact by the cars data. At last, we claim the discriminant analysis is not the inferential statistics because there is no standard errors (SEs) of error rates and discriminant coefficients (Problem4). Therefore, we poroposed the " 100 -fold cross validation for the small sample" method (the method). By this break-through, we can choose the best model having minimum mean of error rate (M2) in the validation sample and obtaine two 95\% confidence intervals (CIs) of error rate and discriminant coefficients. When we discriminate the exam scores by this new method, we obtaine the surprising results seven LDFs except for Fisher's LDF are almost the same as the trivial LDFs. In this research, we discriminate the Japanese 44 cars data because we can discuss four problems. There are six independent variables to discriminate 29 regular cars and 15 small cars. This data is linear separable by the emission rate (X1) and the number of seats (X3). We examine the validity of the new model selection procedure of the discriminant analysis. We proposed the model with minimum mean of error rates (M2) in the validation samples is the best model. We had examined this procedure by the exam scores, and we obtain good results. Moreover, the 95\% CI of eight LDFs offers us real perception of the discriminant theory. However, the exam scores are different from the ordinal data. Therefore, we apply our theory and procedure to the Japanese 44 cars data and confirmed the same conclution.

Keywords: Model Selection Procedure, Means of Error Rates, Fisher's LDF, Logistic Regression, Support Vector Machine (SVM), Minimum Number of Misclassifications (minimum NM, MNM), Revised IP-OLDF based on MNM criterion, Revised IPLP-OLDF, Revised LP-OLDF, Linear Separable Data and Model, K-fold Cross validation.

Corresponding author: Shuichi Shinmura, Prof. (full) / Doctor Science, Economic Department, Seikei Univ. 


\section{Introduction}

In this research, we examine the new model selection procedure of the discriminant analysis by the "k-fold cross validation for small sample" method (the method). Although Lachenbruch et al. [6] had proposed a leave-one-out (LOO) procedure for model selection of the discriminant analysis, they could not achieve the new method because of lack of computer power. If we fix "k=100", we can obtain 100 LDFs and 100 error rates in the training and validation samples. From the 100 error rates, we calculate two means of error rates such M1 and M2 in the training and validation samples. We consider the model with minimum M2 among all possible combination models [5] is the best model. We apply this new procedure for three data sets of exam scores [18] and obtain good results. We had better distinguished these computer-intensive approaches from the traditional inferential statistics with the SE. Genuine statisticians without computer power had established the inferential statistics by their intellectual brain. Now, we can utilize the computer power with statistical and MP solvers such as JMP [7] and LINGO [8] [9]. The researchers who wish to analyze their research data can obtain the $95 \%$ confidence interval (CI) of the error rate and discriminant coefficients [14] [16]. These statistics give us precise and deterministic judgment about the model selection procedure of the discriminant analysis.

\section{Method}

In this research, we discriminate the Japanese 44 cars data (the data) by eight LDFs. We focus on two means of error rates such as "M1 and M2" in the training and validation samples and propose the model with minimum M2s is the best model (the model selection procedure, the procedure). We compare eight M2s of the best model of eight LDFs and the $95 \%$ CI for discriminant coefficients.

\section{Eight LDFs}

In this research, we compare two statistical LDFs and six MP-based LDFs [19] [20]. Two statistical LDFs are Fisher's LDF [2] and a logistic regression [1]. Fisher proposed Fisher's LDF based on the variance-covariance matrices and found the discriminant analysis. After Fisher's LDF, we can use a quadratic discriminant function (QDF). From 1971 to 1974, we analyzed electrocardiogram (ECG) data in order to develop the diagnostic logic between normal and abnormal symptoms by Fisher's LDF and QDF. Our research was inferior to the decision tree logic developed by the medical doctor. After this experience, we found four problems of the discriminant analysis [21][25] and concluded these discriminant functions are fragile for the discrimination of the normal and abnormal diseases. Therefore, most medical researchers used the logistic regression in the equation (1). If some independent variable increases or decreases, the probability $\mathrm{p}$ belongs to class1 (abnormal diseases) increases from 0 (class2) to 1 (class1).

$$
\log (\mathrm{p} /(1-\mathrm{p}))=\mathrm{f}(\mathrm{x})
$$

$\mathrm{p}$ : the probability belongs to class $1 ; \mathrm{x}$ : the independent variables.

We can obtain the maximum/minimum value of the function by MP, regardless of the presence or absence of constraints. Therefore, Schrage [8] introduced several definitions of regression models. Quadratic Programming (QP) defines the ordinal least square method. Linear Programming (LP) defines the "Least Absolute Values (LAV) Regression”. Nonlinear Programming (NLP) represents several Lp norm regression. However, there were few researches about the regression analysis. On the other hands, there were many researches about MP-based discriminant models [46]. However, statistical users rarely used these discriminant functions because 
these functions were not evaluated by the real data. Vapnik [47] proposed three different SVM models examined by the actual data. H-SVM in equation (2) indicates the discrimination of LSD clearly.

$$
\begin{gathered}
\mathrm{MIN}=\|\mathrm{b}\|^{2} / 2 ; \\
\text { yi } *\left({ }^{\mathrm{t}} \mathbf{x} \mathbf{i b}+\mathrm{b} 0\right)>=1 ;
\end{gathered}
$$

$\mathrm{yi}=1 /-1$ for $\mathrm{xi} \in$ class1/class2; $\mathrm{xi}: \mathrm{p}$-independent variables ( $\mathrm{p}$-variables);

$\mathrm{b}: \mathrm{p}$-discriminant coefficients; $\mathrm{b} 0$ : the intercept and free variable.

Real data are rarely linearly separable. For this reason, S-SVM has been defined in equation (3) with two objects. These two objects are combined by defining some "penalty c." However, S-SVM does not have a rule to determine 'c' correctly. In this research, two S-SVMs such as SVM4 (c=10000) and SVM1 (c=1) are examined. We know the "M1 \& M2" of SVM4 are almost better than SVM1.

$$
\mathrm{MIN}=\|\mathbf{b}\|^{2} / 2+\mathrm{c}^{*} \Sigma \mathrm{ei} ; \mathrm{yi} *\left({ }^{\mathrm{t}} \mathbf{x i} \mathbf{b}+\mathrm{b} 0\right)>=1-\mathrm{ei}
$$

c: penalty c; ei: non-negative decision variable.

On the other hand, Shinmura [10-13] [15] developed an revised optimal LDF by Integer Programming (Revised IP-OLDF) based on the minimum NM (MNM) criterion in equation (4) and found several new facts about the discriminant theory. Only Revised IP-OLDF can avoid the cases on the discriminant hyperplane (Problem1). Moreover, only H-SVM and Revised IP-OLDF can recognize linear separable model theoretically. Another LDFs cannot recognize LSD and cannot judge the data is overlap or not theoretically (Problem2).

$$
\mathrm{MIN}=\Sigma \mathrm{ei} ; \mathrm{yi}^{*}\left(\mathrm{t}_{\mathbf{x}} \mathbf{b}+\mathrm{b} 0\right)>=1-\mathrm{M}^{*} \text { ei } ;
$$

ei: 0/1 integer decision variable; $M$ : big $M$ constant ( $M=10000)$; b0: free decision variables.

If ei is a non-negative real variable, we utilize Revised LP-OLDF, which is an L1-norm LDF. Revised IPLP-OLDF is a combined model of Revised LP-OLDF and Revised IP-OLDF [17]. In the first step, Revised LP-OLDF is applied for all cases, and ei fixed to 0 for cases that are discriminated correctly by Revised LP-OLDF. In the second step, Revised IP-OLDF is used for cases, ei of which are not zero in the first step. It is important that ei changes from real decision variable to binary integer variable. For this reason, Revised IPLP-OLDF can be expected to obtain an estimate of MNM faster than Revised IP-OLDF for large samples [22]. On the other hand, Revised IP-OLDF can do feature selection for six microarray data (the datasets) very easy, and find the dataset consists of several small subspaces with $\mathrm{MNM}=0$ and other high-dimension subspaces that is not linear separabe [27-43].

In this research, we compare Revised IP-OLDF with seven LDFs by the "k-fold cross validation for small sample" method. We evaluated Revised IP-OLDF by several small samples. It was difficult for us to compare Revised IP-OLDF with seven LDFs because we could not validate the effectiveness of Revised IP-OLDF. Therefore, we proposed the method and can evaluate eight LDFs by two means of error rates such as M1 and M2. Although Fisher developed Fisher's LDF, he never formulated the equation of SEs of error rates and discriminant coefficients. Therefore, there were no good model selection procedures instead of the LOO method.

In this research, we propose the new model selection procedure as follows:

We discriminate an original data by eight LDFs and two discriminant functions such as QDF and a Regulalized Discriminant Analysis (RDA) [4]. In principal, we analyzed all possible combination models made 


$$
\text { - Japanese } 44 \text { Cars - }
$$

by independent variables. Goodnight established this procedure in the regression analysis by the sweep operator [5]. By this procedure, we can overlook the whole picture of the study.

We discriminate re-sampling samples by the method. In this research, we fix $\mathrm{k}=100$ in order to obtain two means of error rates and $95 \% \mathrm{CI}$ of error rates and discriminant coefficients.

We consider the best model with the minimum values of M2 (minimum M2 standard) and compare eight M2s of eight best models. Although Vapnik defined the generalization ability, we claim the model with minimum M2 has good generalization ability. Moreover, we discuss the $95 \%$ CI of discriminant coefficients.

\section{Forty-four Japanese Car Data}

Let us consider the discrimination of 29 regular cars and 15 small cars having six variables in Table 1. Small cars have a unique Japanese specification. Women buy them as second cars because they cost efficient. The emission rate and capacity (number of seats) of small cars are smaller than the regular cars. The emission rate of small and regular cars ranges from [0.657, 0.658] and [0.996, 3.456], respectively. The seats of small and regular cars are 4 and $[5,8]$, respectively. ' $p$ ' is the number of variables selected by the forward stepwise procedure. QDF and Revised IP-OLDF can find the one variable model (X1: Emission) is linearly separable. Last two columns are NMs of RDA [2]. Before 2012, JMP [5] switched QDF by RDA when QDF found the problems of data. However, both QDF and RDA misclassified all regular cars to the small cars in the three variables because the seats of the small cars are four. If we add little random noise to the constant values, NMs become zero. This problem may be the defect of the generalized inverse matrix technique incremented in QDF of JMP. After this fact, modified RDA was released. We must choose two parameters of $\lambda$ and $\gamma$. We choose the best combination such $\lambda=\gamma=0.1$ by $11 * 11$ trials. Although the best combination is valid for this data, we use these values for another data because we think the survey of best combination is not meaningfull for our research.

Table 1

Comparison of $M N M$ and $N M s$

\begin{tabular}{llllllll}
\hline $\mathrm{p}$ & Var. & $\mathrm{t}$ & LDF & QDF & MNM & $\lambda=\gamma=0.8$ & 0.1 \\
\hline 1 & Emission (X1) & 11.37 & 2 & 0 & 0 & 2 & 0 \\
2 & Price (X2) & 5.42 & 1 & 0 & 0 & 4 & 0 \\
3 & Capacity (X3) & 8.93 & 1 & 29 & 0 & 3 & 0 \\
4 & CO2 (X4) & 4.27 & 1 & 29 & 0 & 4 & 0 \\
5 & Fuel (X5) & -4 & 0 & 29 & 0 & 5 & 0 \\
6 & Sales (X6) & -0.82 & 0 & 29 & 0 & 5 & 0 \\
\hline
\end{tabular}

\section{Results}

\section{The Outlook of Data}

Fig.1 shows the Box-Whisker-Plots of the emission (X1) and capacity (X3). This graph tells us that we can obtain two 1-variable models are linear separable. Therefore, 48 models including (X1) or (X3) are linear separable among 63 models. Other 15 models are not linear separable. 
- Japanese 44 Cars -
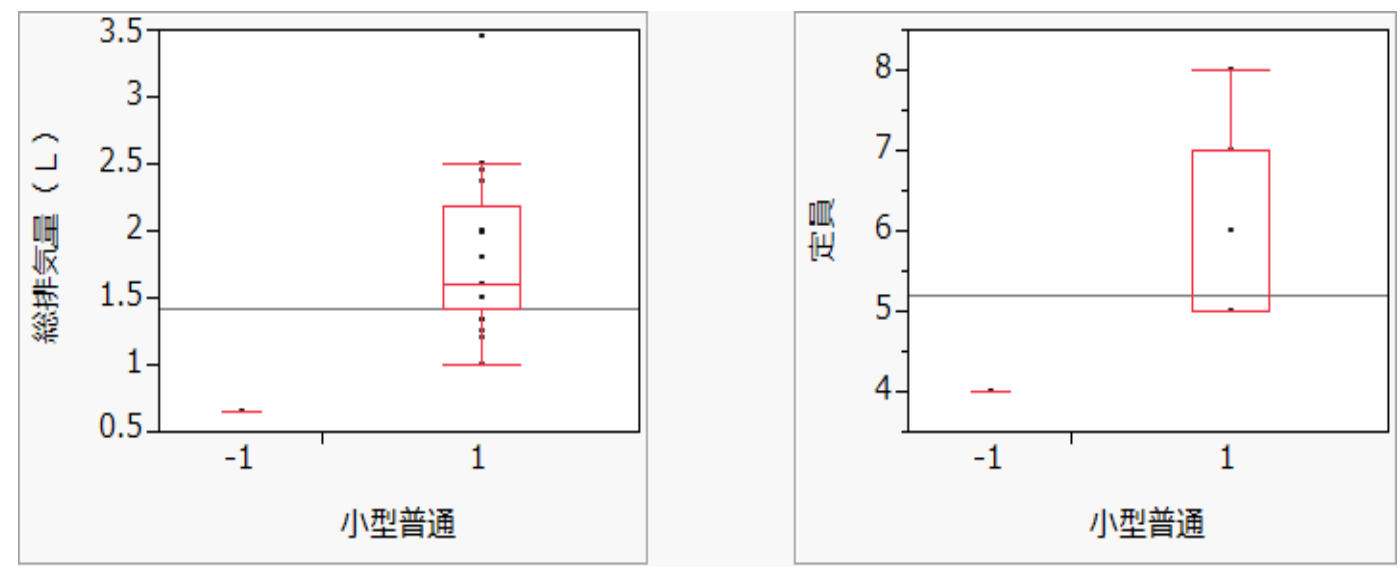

Figure 1. Box-Whisker-Plots of Emission and Capacity (-1: Small Car, 1: Regular Car).

Table2. is the NMs of 48 linear separable models. We categorize these models to three groups. First 16 models from $\mathrm{SN}=1$ to $\mathrm{SN}=16$ include $\mathrm{X} 1$ and $\mathrm{X} 3$. Next 16 models from $\mathrm{SN}=17$ to $\mathrm{SN}=32$ include $\mathrm{X} 1$. Last 16 models from $\mathrm{SN}=33$ to $\mathrm{SN}=48$ include $\mathrm{X} 3$. The NMs of six MP-based LDFs, logistic regression and RDA are zero. Therefore, these results are omitted from the table. On the other hand, Fisher's LDF cannot recognize 41 models those are linear separable. Moreover, QDF misclassified 29 regular cars to the small cars for the first and third groups because these models include X3. If we add little random noise to X3 belonging to the small car class, all NMs become zero. Therefore, we conclude only Fisher's LDF can discriminate seven linear separable models among 48 linear separable models correctly.

Table 2

NMs of eight LDFs by 48 linear separable models

\begin{tabular}{lllllllll}
\hline SN & Emission & Price & Capacity & CO2 & Fuel & Sales & LDF & QDF \\
\hline 1 & 1 & 0 & 1 & 0 & 0 & 0 & 2 & 29 \\
2 & 1 & 1 & 1 & 0 & 0 & 0 & 1 & 29 \\
3 & 1 & 0 & 1 & 1 & 0 & 0 & 1 & 29 \\
4 & 1 & 0 & 1 & 0 & 1 & 0 & 1 & 29 \\
5 & 1 & 0 & 1 & 0 & 0 & 1 & 2 & 29 \\
6 & 1 & 1 & 1 & 1 & 0 & 0 & 1 & 29 \\
7 & 1 & 1 & 1 & 0 & 1 & 0 & 1 & 29 \\
8 & 1 & 1 & 1 & 0 & 0 & 1 & 1 & 29 \\
9 & 1 & 0 & 1 & 1 & 1 & 0 & 1 & 29 \\
10 & 1 & 0 & 1 & 1 & 0 & 1 & 1 & 29 \\
11 & 1 & 0 & 1 & 0 & 1 & 1 & 1 & 29 \\
12 & 1 & 1 & 1 & 1 & 1 & 0 & 0 & 29 \\
13 & 1 & 1 & 1 & 1 & 0 & 1 & 0 & 29 \\
14 & 1 & 1 & 1 & 0 & 1 & 1 & 1 & 29 \\
15 & 1 & 0 & 1 & 1 & 1 & 1 & 1 & 29 \\
16 & 1 & 1 & 1 & 1 & 1 & 1 & 0 & 29 \\
17 & 1 & 0 & 0 & 0 & 0 & 0 & 2 & 0 \\
18 & 1 & 1 & 0 & 0 & 0 & 0 & 1 & 0 \\
19 & 1 & 0 & 0 & 1 & 0 & 0 & 1 & 0 \\
20 & 1 & 0 & 0 & 0 & 0 & 1 & 2 & 0 \\
21 & 1 & 0 & 0 & 0 & 1 & 0 & 2 & 0 \\
\hline
\end{tabular}


Table 2 continued

\begin{tabular}{|c|c|c|c|c|c|c|c|c|}
\hline SN & Emission & Price & Capacity & $\mathrm{CO} 2$ & Fuel & Sales & LDF & QDF \\
\hline 22 & 1 & 1 & 0 & 1 & 0 & 0 & 1 & 0 \\
\hline 23 & 1 & 1 & 0 & 0 & 1 & 0 & 1 & 0 \\
\hline 24 & 1 & 1 & 0 & 0 & 0 & 1 & 1 & 0 \\
\hline 25 & 1 & 0 & 0 & 1 & 1 & 0 & 1 & 0 \\
\hline 26 & 1 & 0 & 0 & 1 & 0 & 1 & 1 & 0 \\
\hline 27 & 1 & 0 & 0 & 0 & 1 & 1 & 4 & 0 \\
\hline 28 & 1 & 1 & 0 & 1 & 1 & 0 & 0 & 0 \\
\hline 29 & 1 & 1 & 0 & 1 & 0 & 1 & 1 & 0 \\
\hline 30 & 1 & 1 & 0 & 0 & 1 & 1 & 1 & 0 \\
\hline 31 & 1 & 0 & 0 & 1 & 1 & 1 & 2 & 0 \\
\hline 32 & 1 & 1 & 0 & 1 & 1 & 1 & 0 & 0 \\
\hline 33 & 0 & 0 & 1 & 0 & 0 & 0 & 0 & 29 \\
\hline 34 & 0 & 1 & 1 & 0 & 0 & 0 & 5 & 29 \\
\hline 35 & 0 & 0 & 1 & 0 & 1 & 0 & 3 & 29 \\
\hline 36 & 0 & 0 & 1 & 0 & 0 & 1 & 1 & 29 \\
\hline 37 & 0 & 0 & 1 & 1 & 0 & 0 & 0 & 29 \\
\hline 38 & 0 & 1 & 1 & 1 & 0 & 0 & 5 & 29 \\
\hline 39 & 0 & 1 & 1 & 0 & 1 & 0 & 6 & 29 \\
\hline 40 & 0 & 1 & 1 & 0 & 0 & 1 & 6 & 29 \\
\hline 41 & 0 & 0 & 1 & 1 & 1 & 0 & 3 & 29 \\
\hline 42 & 0 & 0 & 1 & 0 & 1 & 1 & 3 & 29 \\
\hline 43 & 0 & 0 & 1 & 1 & 0 & 1 & 1 & 29 \\
\hline 44 & 0 & 1 & 1 & 1 & 1 & 0 & 4 & 29 \\
\hline 45 & 0 & 1 & 1 & 1 & 0 & 1 & 5 & 29 \\
\hline 46 & 0 & 1 & 1 & 0 & 1 & 1 & 6 & 29 \\
\hline 47 & 0 & 0 & 1 & 1 & 1 & 1 & 4 & 29 \\
\hline 48 & 0 & 1 & 1 & 1 & 1 & 1 & 5 & 29 \\
\hline
\end{tabular}

\section{Comparison of fifteen Discriminant Function}

Table 3 shows the NMs, and the number of cases of the discriminant-hyperplane $f(x i)=0$ by 15 non-linear separable models. Four variables correspond to four columns. Because these models do not include X1 and X3, these two variables are omitted from the table. Sixth columns is MNM. Next eight columns are Diffs of eight discriminant functions. 'Diff' is the difference of ( eight NM - MNM). We omit the NM of H-SVM because it cannot discriminate these models. Last column is the number of cases on $\mathrm{f}(\mathrm{xi})=0$ of Revised LP-OLDF. Other LDFs are free from Problem1 for this data.

Table 3

Diffs and the number on $f(x)=0$ by 15 non-linear separable models

\begin{tabular}{lllllllllllllll}
\hline \multicolumn{1}{c}{ Variable } & \multicolumn{1}{c}{ MNM } & \multicolumn{1}{c}{ Diff } & & & $\mathrm{f}(\mathrm{xi})=0$ \\
\hline SN & X2 & X4 & X5 & X6 & RIP & SVM4 & SVM1 & LP & IPLP & Logistic & LDF & QDF & RDA & LP \\
\hline 49 & 1 & 0 & 0 & 0 & 5 & 1 & 1 & 1 & 0 & 0 & 8 & 2 & 7 & 0 \\
50 & 0 & 0 & 1 & 0 & 10 & 1 & 1 & 1 & 0 & 1 & 1 & 2 & 2 & 1 \\
51 & 0 & 1 & 0 & 0 & 10 & 1 & 1 & 1 & 0 & 1 & 3 & 2 & 2 & 1 \\
52 & 0 & 0 & 0 & 1 & 13 & 2 & 2 & 2 & 2 & 1 & 2 & 1 & 2 & 0 \\
\hline
\end{tabular}


Table 3 continued

\begin{tabular}{lllllllllllllll}
\hline \multicolumn{1}{c}{ Variable } & \multicolumn{1}{c}{ MNM } & \multicolumn{1}{c}{ Diff } & \multicolumn{1}{c}{$\mathrm{f}(\mathrm{xi})=0$} \\
\hline SN & X2 & X4 & X5 & X6 & RIP & SVM4 & SVM1 & LP & IPLP & Logistic & LDF & QDF & RDA & LP \\
\hline 53 & 1 & 0 & 1 & 0 & 4 & 2 & 2 & 2 & 0 & 1 & 4 & 3 & 6 & 0 \\
54 & 1 & 1 & 0 & 0 & 4 & 2 & 2 & 2 & 0 & 2 & 7 & 4 & 8 & 0 \\
55 & 0 & 0 & 1 & 1 & 8 & 6 & 6 & 6 & 1 & 3 & 3 & 1 & 1 & 0 \\
56 & 1 & 0 & 0 & 1 & 4 & 2 & 2 & 2 & 0 & 1 & 8 & 3 & 8 & 0 \\
57 & 0 & 1 & 1 & 0 & 10 & 2 & 2 & 1 & 0 & 2 & 1 & 4 & 1 & 1 \\
58 & 0 & 1 & 0 & 1 & 8 & 3 & 3 & 3 & 0 & 4 & 5 & 4 & 4 & 0 \\
59 & 1 & 0 & 1 & 1 & 4 & 2 & 2 & 2 & 0 & 1 & 4 & 3 & 6 & 0 \\
60 & 1 & 1 & 1 & 0 & 4 & 0 & 0 & 0 & 0 & 0 & 5 & 5 & 9 & 0 \\
61 & 1 & 1 & 0 & 1 & 4 & 2 & 2 & 2 & 0 & 1 & 7 & 4 & 7 & 0 \\
62 & 0 & 1 & 1 & 1 & 8 & 7 & 7 & 7 & 0 & 3 & 2 & 2 & 3 & 0 \\
63 & 1 & 1 & 1 & 1 & 3 & 1 & 1 & 1 & 0 & 1 & 6 & 5 & 9 & 0 \\
\hline
\end{tabular}

\section{The 100-fold Cross validation Method}

In this chapter, we compare six MP-based LDFs and two statistical LDFs by the method. In this research, we examine seven models made by three variables such as $\mathrm{X} 1, \mathrm{X} 2$, and $\mathrm{X} 3$ because other three variables are not important for the discrimination explained by Table1. Therefore, there are six linear separable models including X1 or X3, and one 1-variable model (X2) that is not linear separable. Table 4 shows the results by the method. We omit QDF and RDA because those are not LDFs.

We examine seven discriminant models of seven LDFs and six models of H-SVM. First seven rows are seven discriminant models of Revised IP-OLDF (RIP). 'Model' column shows the independent variable. M1s and M2s are the mean of error rates in the training and validation samples. Six M1s and M2s of Revised LP-OLDF are zero. Six M1s and five M2s of Revised IP-OLDF and Revised IPLP-OLDF are zero. Six M1s and three M2s of HSVM, SVM4 and logistic regression are zero. Only two M1s and M2s of SVM1 are zero. All M1s and M2s of Fisher's LDF are not zero. We can summarize these results about six linear separable models as follows:

We can roughly evaluate the ranking of eight LDFs as follows. Revised LP-OLDF is the first rank. Revised IP-OLDF and Revised IPLP-OLDF are the second rank. HSVM, SVM4 and logistic regression are the third rank. Although SVM1 and Fisher's LDF are the fourth and fifth rank, these cannot recognize the linear separable models.

We can choose the fifth and sixthe models are the best model because of two reasons. Although there are several models having "minimum M2 $=0$ ", we choose these models by the principal parsimony because those are 1-variable model. Revised IP-OLDF, SVM4, Revised LP-OLDF, Revised IPLP-OLDF and logistic regression choose these two models as the best models.

Seventh model is not linear separable. Six values of 'M2Diff.' are 27.89, 0.23, 0.23, 0.02, 0.26 and 14.28\%, respectively. SVM4 and Fisher's LDF are $27.89 \%$ and $14.28 \%$ worse than Revised IP-OLDF. Although the discrimination of one variable is not important, we must investigate the reason why these two results are so bad. On the other hand, absolute values of other LDFs are within $0.26 \%$. In the pass/fail determinations [18][19], Revised IP-OLDF is superior to other seven LDFs for the linear separable data. We confirm these facts in this data. 
Table 4

M1s and M2s of eight LDFs.

\begin{tabular}{|c|c|c|c|c|c|c|}
\hline \multirow{8}{*}{$\begin{array}{l}\text { MNM } \\
50 \mathrm{~s}\end{array}$} & & M1 & M2 & Diff2. & Model & \\
\hline & 1 & 0 & 0 & 0 & $\mathrm{X} 1, \mathrm{X} 2, \mathrm{X} 3$ & \\
\hline & 2 & 0 & 0 & 0 & $\mathrm{X} 1, \mathrm{X} 2$ & \\
\hline & 3 & 0 & 0.07 & 0.07 & $\mathrm{X} 1, \mathrm{X} 3$ & \\
\hline & 4 & 0 & 0 & 0 & $\mathrm{X} 2, \mathrm{X} 3$ & \\
\hline & 5 & 0 & 0 & 0 & $\mathrm{X} 1$ & \\
\hline & 6 & 0 & 0 & 0 & $\mathrm{X} 3$ & \\
\hline & 7 & 9.55 & 12.75 & 3.2 & $\mathrm{X} 2$ & \\
\hline SVM4 & & M1 & M2 & Diff2. & M1Diff & M2Diff \\
\hline $40 \mathrm{~s} 1$ & & 0 & 0.11 & 0.11 & 0 & 0.11 \\
\hline 2 & & 0 & 0.2 & 0.2 & 0 & 0.2 \\
\hline 3 & & 0 & 0 & 0 & 0 & -0.07 \\
\hline 4 & & 0 & 0.11 & 0.11 & 0 & 0.11 \\
\hline 5 & & 0 & 0 & 0 & 0 & 0 \\
\hline 6 & & 0 & 0 & 0 & 0 & 0 \\
\hline 7 & & 40.45 & 40.64 & 0.18 & 30.91 & 27.89 \\
\hline $\begin{array}{l}\text { SVM1 } \\
\end{array}$ & & M1 & M2 & Diff2. & M1Diff & M2Diff \\
\hline $44 \mathrm{~S} 1$ & & 1.14 & 1.2 & 0.07 & 1.14 & 1.2 \\
\hline 2 & & 0.98 & 1.7 & 0.73 & 0.98 & 1.7 \\
\hline 3 & & 0 & 0 & 0 & 0 & -0.07 \\
\hline 4 & & 0.34 & 0.5 & 0.16 & 0.34 & 0.5 \\
\hline 5 & & 0.73 & 0.84 & 0.11 & 0.73 & 0.84 \\
\hline 6 & & 0 & 0 & 0 & 0 & 0 \\
\hline 7 & & 12.39 & 12.98 & 0.59 & 2.84 & 0.23 \\
\hline LP & & M1 & M2 & Diff2. & M1Diff & M2Diff \\
\hline 1 & & 0 & 0 & 0 & 0 & 0 \\
\hline 2 & & 0 & 0 & 0 & 0 & 0 \\
\hline 3 & & 0 & 0 & 0 & 0 & -0.07 \\
\hline 4 & & 0 & 0 & 0 & 0 & 0 \\
\hline 5 & & 0 & 0 & 0 & 0 & 0 \\
\hline 6 & & 0 & 0 & 0 & 0 & 0 \\
\hline 7 & & 12.39 & 12.98 & 0.59 & 2.84 & 0.23 \\
\hline IPLP & & M1 & M2 & Diff2. & M1Diff & M2Diff \\
\hline $1 \mathrm{~m} 40 \mathrm{~s} 1$ & & 0 & 0.27 & 0.27 & 0 & 0.27 \\
\hline 2 & & 0 & 0 & 0 & 0 & 0 \\
\hline 3 & & 0 & 0 & 0 & 0 & -0.07 \\
\hline 4 & & 0 & 0 & 0 & 0 & 0 \\
\hline 5 & & 0 & 0 & 0 & 0 & 0 \\
\hline 6 & & 0 & 0 & 0 & 0 & 0 \\
\hline 7 & & 9.55 & 12.77 & 3.23 & 0 & 0.02 \\
\hline HSVM & & M1 & M2 & Diff2. & M1Diff & M2Diff \\
\hline 1 & & 0 & 0.11 & 0.11 & 0 & 0.11 \\
\hline 2 & & 0 & 0.2 & 0.2 & 0 & 0.2 \\
\hline 3 & & 0 & 0 & 0 & 0 & -0.07 \\
\hline 4 & & 0 & 0.11 & 0.11 & 0 & 0.11 \\
\hline 5 & & 0 & 0 & 0 & 0 & 0 \\
\hline 6 & & 0 & 0 & 0 & 0 & 0 \\
\hline
\end{tabular}


Table 4 to be continued

\begin{tabular}{llllll}
\hline Logi & M1 & M2 & Diff 2 & M1Diff & M2Diff \\
\hline 1 & 0 & 0.36 & 0.36 & 0 & 0.36 \\
2 & 0 & 0.05 & 0.05 & 0 & 0.05 \\
3 & 0 & 0.02 & 0.02 & 0 & -0.05 \\
4 & 0 & 0 & 0 & 0 & 0 \\
5 & 0 & 0 & 0 & 0 & 0 \\
6 & 0 & 0 & 0 & 0 & 0.26 \\
7 & 12.3 & 13.01 & 0.72 & 2.75 & M2Diff \\
\hline LDF & M1 & M2 & Diff 2 & 1.5 & 2.35 \\
2 & 1.5 & 2.35 & 0.85 & 1.89 & 4.91 \\
3 & 1.89 & 2.91 & 1.03 & 4.52 & 12.83 \\
4 & 4.52 & 4.75 & 0.23 & 10.43 & 5.74 \\
5 & 10.43 & 12.83 & 2.4 & 5.36 & 6.09 \\
7 & 5.36 & 5.74 & 0.38 & 4.7 & 14.28 \\
\hline
\end{tabular}

\section{The 95\% CI of Discriminant Coefficients by six MP-based LDFs}

Table 5 shows the $95 \%$ CI of eight LDFs. Because all intercepts fix to one, those are omitted from the table.

(1) Revised IP-OLDF

The equation (5) is the full model of Revised IP-OLDF showed by the median. Although the $95 \%$ CI of X3 is $[-0.2222,-0.2222]$ and the constant, we guess this result caused by the "X3=4" of the small car. If X3 of the small car vary, the $95 \% \mathrm{CI}$ is not the constant. Because both coefficients of X1 and X2 are zero, Revised IP-OLDF tells us that X1 and X2 are not important for the discrimination of this data. Although we choose two best models by the minimum M2 standard, we can judge the best model is the fifthe or sixth model. Moreover, the forward stepwise procedure and t-test chose the X1 at first in the Table1. We claim the statistical suggestions are different from the $95 \% \mathrm{CI}$ of coefficients. The equation (5) means the discriminant hyperplane is $\mathrm{X} 3=4.504505$. If $\mathrm{X} 3<4.5$, we can judge the car belongs to the small car. Otherwise, if $\mathrm{X} 3>4.5$, we can judge the car belongs to the regular car. This discriminant rule is as same as the fact that the small cars have four seats and the regular cars have over five seats.

$$
\text { RIP123: } 0^{*} \times \mathrm{X} 1+0 * \times \mathrm{X} 2-0.2222 * \times \mathrm{X} 3+1=-0.2222 * \times \mathrm{X} 3+1=0
$$

The equations (6) is 2-variables models such as $(\mathrm{X} 1, \mathrm{X} 2)$. Because the emission rate of small and regular cars ranges from $[0.657,0.658]$ and $[0.996,3.456]$, we can judge this model is linear separable by X1 without X2. The discriminant hyperplane is $\mathrm{X} 1=0.82713$. The $95 \% \mathrm{CI}$ of $\mathrm{X} 1$ in 1 -variable model (X1) has almost the same $95 \% \mathrm{CI}$ of fifth model. Both equations (5) and (6) tell us X3 is more important than X1 for the discrimination this data is linear separable.

$$
\text { RIP12: }-1.209 * \times \mathrm{X} 1+0 * \times \mathrm{X} 2+1=-1.209 * \times \mathrm{X} 1+1=0
$$

The equations (7) is 2-variables models such as (X1, X3). Although each variable recognize the linear separable model, both coefficients of $(\mathrm{X} 1, \mathrm{X} 3)$ are zero at the $5 \%$ level. We guess each variable disturbs other variable and investigate this fact in near future.

$$
\text { RIP13: }-1.078 \times \mathrm{X} 1+0 \times \mathrm{X} 3=0
$$

The equations (8) are 2-variables models such as (X2, X3). The $95 \% \mathrm{CI}$ of $\mathrm{X} 3$ has the same $95 \% \mathrm{CI}$ of $\mathrm{X} 3$ in the full model, and 1-variable model (X3). 


$$
\text { - Japanese } 44 \text { Cars - }
$$

$$
\text { RIP23: } 0 * \times \mathrm{X} 2-0.2222 * \times \mathrm{X} 3+1=-0.2222 * \times \mathrm{X} 3+1=0
$$

The equation (9) is 1 -variable model such as (X2). The discriminant hyperplane is $\mathrm{X} 2=1333333$. The prices of four regular cars are less than 1,333 thousand yen and those of two small cars are higher than this price. Therefore, the error rate is 0.090909 .

$$
\text { RIP2: }-0.00000075 * \times \mathrm{X} 2+1=0
$$

(2) Comparison of H-SVM and Revised IP-OLDF

Fourth, sixth and fifth models of H-SVM and Revised IP-OLDF are the same. However, first, second and third models of both LDFs are quiet different. This facts tells us as follows: Because H-SVM fixes some cases on two support vectors (SVs), three coefficients of X1, X2, and X3 of H-SVM have ranges such as [-0.11, -0.071$]$, [-7.2E-8, 4.5E-8], [-0.218. -0.177], respectively. On the other hand, although Revised IP-OLDF has two SVs, it does not take effect of SVs and 100 LDFs become the same equation (5).

\begin{tabular}{|c|c|c|c|c|c|c|c|c|}
\hline & RIP & $\mathrm{X} 1$ & $\mathrm{X} 2$ & $\mathrm{X} 3$ & H-SVM & $\mathrm{X} 1$ & $\mathrm{X} 2$ & X3 \\
\hline & $97.50 \%$ & 0 & 0 & -0.222 & $97.5 \%$ & -0.071 & 4.50E-08 & $-1.77 \mathrm{E}-01$ \\
\hline 1 & Median & 0 & 0 & -0.222 & Median & -0.073 & 0 & -0.209 \\
\hline & $2.50 \%$ & 0 & 0 & -0.222 & $2.5 \%$ & -0.116 & $-7.20 \mathrm{E}-08$ & -0.218 \\
\hline \multirow{3}{*}{2} & $97.50 \%$ & -1.053 & 0 & & $97.5 \%$ & -0.5115 & $9.62 \mathrm{E}-07$ & \\
\hline & Median & -1.209 & 0 & & Median & -1.2092 & 0 & \\
\hline & $2.50 \%$ & -2.184 & 0 & & $2.5 \%$ & -2.4382 & $-3.50 \mathrm{E}-07$ & \\
\hline \multirow{3}{*}{3} & $97.50 \%$ & 379174 & & 0 & $97.5 \%$ & -0.0707 & & -0.1978 \\
\hline & Median & -1.0776 & & 0 & Median & -0.0707 & & -0.2092 \\
\hline & $2.50 \%$ & -1.2092 & & -74249 & $2.5 \%$ & -0.1155 & & -0.2092 \\
\hline \multirow{3}{*}{4} & $97.50 \%$ & & 0 & -0.222 & $97.5 \%$ & & $0.00 \mathrm{E}+00$ & -0.222 \\
\hline & Median & & 0 & -0.222 & Median & & $0.00 \mathrm{E}+00$ & -0.222 \\
\hline & $2.50 \%$ & & 0 & -0.222 & $2.5 \%$ & & $0.00 \mathrm{E}+00$ & -0.222 \\
\hline \multirow{3}{*}{5} & $97.50 \%$ & -1.053 & & & $97.5 \%$ & -1.053 & & \\
\hline & Median & -1.209 & & & Median & -1.209 & & \\
\hline & $2.50 \%$ & -1.209 & & & $2.5 \%$ & -1.209 & & \\
\hline \multirow{3}{*}{6} & $97.50 \%$ & & & -0.222 & $97.5 \%$ & & & -0.222 \\
\hline & Median & & & -0.222 & Median & & & -0.222 \\
\hline & $2.50 \%$ & & & -0.222 & $2.5 \%$ & & & -0.222 \\
\hline \multirow{3}{*}{7} & $97.50 \%$ & & -6.7 & & & & & \\
\hline & Median & & -7.5 & & & & & \\
\hline & $2.50 \%$ & & -8.2 & & & & & \\
\hline
\end{tabular}

Table 5

The 95\% confidence interval (CI) of eight LDFs. 


$$
\text { - Japanese } 44 \text { Cars - }
$$

\section{The $\mathbf{9 5 \%}$ CI of Discriminant Coefficients by Fisher's LDF and logistic regression}

Table 6 shows the 95\% CI of Fisher's LDF and logistic regression. We suppose the yi as the object variable and analyze the data by the regression analysis because the obtained regression coefficients are propotinal to the Fisher's LDF by the plug-in rule. First row is the coefficients. Second raw is the standard error (SE). Third row is the p-value by t-test. We know only coefficient of X2 in the fourth model (X2, X3) is zero. Another coefficients are rejected at $5 \%$ or $1 \%$ levels. Therefore, it is difficult for us to choose good model among six models. On the other hand, we know all coefficient of six linear separable logistic regression models are zero [3]. Because seventh model is not linear separable, this 1-variable model (X2) is rejected at $1 \%$ level. We had better not trusted the SE and p-value of Fisher's LDF by the plug-in rule. In addition to this recommendation, we cannot use the SE and p-valu of logistic regression.

Table 6

The 95\% CI of Fisher's LDF and logistic regression.

\begin{tabular}{|c|c|c|c|c|c|c|c|c|c|c|}
\hline & LDF & $\mathrm{X} 1$ & X2 & X3 & c & logistic & $\mathrm{X} 1$ & $\mathrm{X} 2$ & X3 & $\mathrm{c}$ \\
\hline \multirow{3}{*}{1} & Coeff. & 1.84 & $-7.90 \mathrm{E}-07$ & 0.179 & -1.709 & Coeff. & -19.488 & $6.93 \mathrm{E}-06$ & $-5.37 \mathrm{E}+01$ & 251.697 \\
\hline & SE & 0.25 & $1.56 \mathrm{E}-07$ & 0.08 & 0.32 & $\mathrm{SE}$ & 0 & 2.317 & 2979878 & 1283091 \\
\hline & $\mathrm{P}$ & 0.0001 & 0.0001 & 0.031 & 0.0001 & $\mathrm{P}$ & . & 1 & 1 & 1 \\
\hline \multirow{3}{*}{2} & Coeff. & 2.119 & $-8.41 \mathrm{E}-07$ & & -1.085 & Coeff. & -179.039 & $1.75 \mathrm{E}-03$ & & 87.858 \\
\hline & SE & 0.227 & $1.62 \mathrm{E}-07$ & & 0.164 & SE & 0 & 1.774 & & 2497413 \\
\hline & $\mathrm{P}$ & 0.0001 & 0.0001 & & 0.0001 & $\mathrm{P}$ & . & 1 & & 1 \\
\hline \multirow{3}{*}{3} & Coeff. & 0.78 & & 0.235 & -2.003 & Coeff. & -3.777 & & -34.414 & 158.844 \\
\hline & SE & 0.175 & & 0.101 & 0.399 & SE & 11909 & & 8185.144 & 27565 \\
\hline & $\mathrm{P}$ & 0.0001 & & 0.024 & 0.0001 & $\mathrm{P}$ & 0.9997 & & 0.9966 & 0.9954 \\
\hline \multirow{3}{*}{4} & Coeff. & & 1.62E-07 & 0.474 & -2.443 & Coeff. & & $-2.77 \mathrm{E}-07$ & -62.889 & 283.495 \\
\hline & SE & & $1.31 \mathrm{E}-07$ & 0.105 & 0.46 & SE & & $2.12 \mathrm{E}+00$ & 2721988 & 11568553 \\
\hline & $\mathrm{P}$ & & 0.222 & 0.0001 & 0.0001 & $\mathrm{P}$ & & 1 & 1 & 1 \\
\hline \multirow{3}{*}{5} & Coeff. & 1.068 & & & -1.191 & Coeff. & -94.471 & & & 79.66 \\
\hline & SE & 0.131 & & & 0.207 & SE & 6289 & & & 5492.898 \\
\hline & $\mathrm{P}$ & 0.0001 & & & 0.0001 & $\mathrm{P}$ & 0.988 & & & 0.988 \\
\hline \multirow{3}{*}{6} & Coeff. & & & 0.549 & -2.528 & Coeff. & & & -37.05 & 166.806 \\
\hline & $\mathrm{SE}$ & & & 0.086 & 0.458 & SE & & & 3702.95 & 17078 \\
\hline & $\mathrm{P}$ & & & 0.0001 & 0.0001 & $\mathrm{P}$ & & & 0.992 & 0.992 \\
\hline \multirow{3}{*}{7} & Coeff. & & $5.04 \mathrm{E}+00$ & & -0.636 & Coeff. & & $-6.97 \mathrm{E}-06$ & & 9.233 \\
\hline & $\mathrm{SE}$ & & $1.29 \mathrm{E}-07$ & & 0.273 & $\mathrm{SE}$ & & $2.60 \mathrm{E}-06$ & & 3.421 \\
\hline & $\mathrm{P}$ & & $3.00 \mathrm{E}-04$ & & 0.025 & $\mathrm{P}$ & & $7.30 \mathrm{E}-03$ & & 0.007 \\
\hline
\end{tabular}




\section{Consideration of the Statistical Analysis}

Fig. 2 is score plots by the PCA by six independent variables. X-axis is first principal component and Y-axis are second and third principal components. The left small $99 \%$ probability ellipses are the small cars plotted by the symbol ' $\therefore$. The right large $99 \%$ probability ellipses are the regular cars plotted by the symbol ' + '. Although this data is linear separable, the regular car ellipses include the small car.
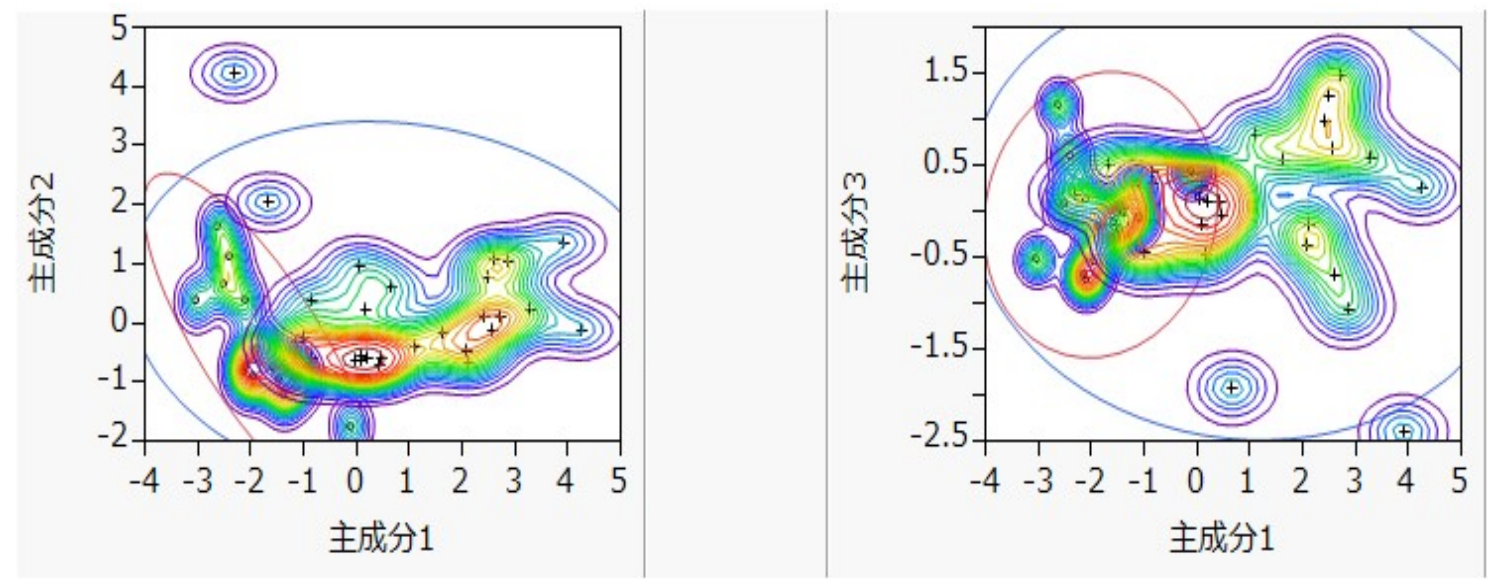

Figure 2. Score Plots by the principal components analysis (PCA).

If we use the indicator yi as the dependent variable and analyze the car data by the regression analysis, the obtained regression coefficients are proportional to the discriminant coefficients by the plug-in rule. Therefore, we can use the model selection procedures and statistics of the regression analysis. Table 7 is a summary of all possible combinations of six variables. We sort six 1-variable models in descending order by R-squares. After 2-variables model, the forward stepwise procedure selects these five models. AIC and BIC recommend fifth model, and Cp statistics suggests full mode. Because our research suggests 1-variable model (X3), we must examine whether we can use these statistics for the discriminant analysis in near future.

Table 7

A summary of all possible combinations of six variables

\begin{tabular}{lllllllll}
\hline $\mathrm{p}$ & Model & R-square & RMSE & AICc & BIC & Cp & RIP & LDF \\
\hline 1 & X1 & 0.61 & 0.3 & 24.2 & 28.9 & 47.8 & 0 & 2 \\
1 & X3 & 0.49 & 0.35 & 35.9 & 40.7 & 74.8 & 0 & 0 \\
1 & X2 & 0.27 & 0.42 & 52 & 56.8 & 125.5 & 6 & 13 \\
1 & X5 & 0.25 & 0.42 & 53.1 & 57.8 & 129.4 & 11 & 11 \\
1 & X4 & 0.23 & 0.42 & 54 & 58.8 & 133.2 & 12 & 13 \\
1 & X6 & 0.02 & 0.48 & 65 & 69.8 & 182.4 & 15 & 15 \\
2 & X1, X2 & 0.77 & 0.24 & 4.3 & 10.4 & 14.9 & 0 & 1 \\
3 & X1-X3 & 0.79 & 0.23 & 1.7 & 9 & 11 & 0 & 1 \\
4 & X1-X4 & 0.82 & 0.22 & -1.4 & 7 & 7.3 & 0 & 1 \\
5 & X1-X5 & 0.84 & 0.21 & -3.4 & $\underline{6}$ & 5 & 0 & 0 \\
6 & X1-X6 & 0.84 & 0.21 & -0.4 & 9.8 & $\underline{7}$ & 0 & 0 \\
\hline
\end{tabular}




\section{Conclusion}

In this research, we discuss the new model selection procedure of the discriminant analysis. We discriminate the Japanese 44 cars data. Although AIC and BIC suggest 5-variables model and Cp suggests full model by the regression analysis, the "M2 minimum standard" procedure recommends 1-variable model (X3). Revised IP-OLDF, H-SVM, SVM4, SVM1, Revised IPLP-OLDF and logistic regression support this model. Moreover, the $95 \% \mathrm{CI}$ of the discriminant coefficients suggest the best LDF as $\mathrm{f}(\mathrm{x} 3)=-0.2222 * \mathrm{X} 3+1$. The discriminant scores of the fifteen small cars are -0.8888 and the scores of the twenty-nine regular cars are greater than 1.111. This fact tells us that we need not discriminate the car data by the discriminant analysis. We can obtain clear judgment by looking at the distribution of six variables.

\section{References}

[1]. Cox, DR., (1959). "The regression analysis of binary sequences (with discussion," Journal Royal Statistics Society, B 20, pp.215-242.

[2]. Fisher, R. A., (1936). The Use of Multiple Measurements in Taxonomic Problems. Annals of Eugenics, 7, 179-188.

[3]. B. Flury and H. Rieduyl, “Multivariate Statistics: A Practical Approach,” Cambridge University Press, 1988.

[4]. Friedman, J. H., (1989). Regularized Discriminant Analysis. Journal of the American Statistical Association, 84/405, 165-175.

[5]. Goodnight, J.H., (1981). A tutorial on the SWEEP Operator. The American Statistician, 33, 149-158.

[6]. Lachenbruch, P. A., Mickey, M. R., (1968). Estimation of error rates in discriminant analysis. Technometrics, 10, 1-11.

[7]. Sall, J. P., Creighton, L., Lehman, A., (2004). JMP Start Statistics, Third Edition. SAS Institute Inc.

[8]. Schrage, L., (1991). LINDO -An Optimization Modeling System (Fourth Edition)-. The Scientific Press.

[9]. Schrage, L., (2006). Optimization Modeling with LINGO. LINDO Systems Inc.

[10]. Shinmura, S., (1998). Optimal Linear Discriminant Functions using Mathematical Programming. Journal of the Japanese Society of Computer Statistics, 11 / 2, 89-101.

[11]. Shinmura, S., (2000). A new algorithm of the linear discriminant function using integer programming. New Trends in Probability and Statistics, 5, 133-142.

[12]. Shinmura, S., (2004). New Algorithm of Discriminant Analysis using Integer Programming. IPSI 2004 Pescara VIP Conference CD-ROM, 1-18.

[13]. Shinmura, S., (2007a). Comparison of Revised IP-OLDF and SVM. ISI2009, 1-4.

[14]. Shinmura, S., (2007b). Overviews of Discriminant Function by Mathematical Programming. Journal of the Japanese Society of Computer Statistics, 20/1-2, 59-94.

[15]. Shinmura, S., (2009). Practical discriminant analysis by IP-OLDF and IPLP-OLDF. IPSI 2009 Belgrade VIPSI Conference CD-ROM, 1-17.

[16]. Shinmura, S., (2010a). The optimal linear discriminant function. Union of Japanese Scientist and Engineer Publishing.

[17]. Shinmura, S., (2010b). Improvement of CPU time of Revised IP-OLDF using Linear Programming. Journal of the Japanese Society of Computer Statistics, 22/1, 39-57.

[18]. Shinmura, S., (2011a).Problems of Discriminant Analysis by Mark Sense Test Data.Japanese Society of Applied Statistics, 40/3, 157-172.

[19]. Shinmura, S., (2011b). Beyond Fisher's Linear Discriminant Analysis - New World of Discriminant Analysis -. ISI2011 CD-ROM, 1-6.

[20]. Shinmura, S., (2013). Evaluation of Optimal Linear Discriminant Function by 100-fold Cross-validation. 2013 ISI CD-ROM, 1-6.

[21]. Shinmura, S., (2014a). End of Discriminant Functions based on Variance-Covariance Matrices. ICORES, 5-14, 2014.

[22]. Shinmura, S., (2014b). Improvement of CPU time of Linear Discriminant Functions based on MNM criterion by IP. Statistics, Optimization and Information Computing, 2, 14-129.

[23]. Shinmura, S., (2014c). Comparison of Linear Discriminant Function by K-fold Cross-validation. Data Analytic 2014, 1-6. 


\section{- Japanese 44 Cars -}

[24]. Shinmura, S., (2015a). The 95\% confidence intervals of error rates and discriminant coefficients. Statistics, Optimization and Information Computing, 3, 66-78.

[25]. Shinmura, S., (2015b). Four Serious Problems and New Facts of the Discriminant Analysis. In Pinson, E., Valente, F., Vitoriano, B., (Eds.), Operations Research and Enterprise Systems, 15-30, Springer (ISSN: 1865-0929, ISBN: 978-3-319-17508-9, DOI: 10.1007/978-3-319-17509-6).

[26]. Shinmura, S., (2015). “A Trivial Linear Discriminant Function. Statistics," Optimization, and Information Computing, Vol.3, pp. 322-335. DOI: 10.19139/soic.20151202.

[27]. Shinmura, S., (2015). The Discrimination of the microarray data (Ver. 1). Research Gate (1), Oct. 28, 2015 , pp. 1-4.

[28]. Shinmura, S., (2015). Feature Selection of three Microarray data. Research Gate (2), Nov.1, 2015, pp. 1-7.

[29]. Shinmura, S., (2015). Feature Selection of Microarray Data (3) - Shipp et al. Microarray Data. Research Gate (3), 2015, pp. $1-11$.

[30]. Shinmura, S., (2015). Validation of Feature Selection (4) - Alon et al. Microarray Data. Research Gate (4), 2015, pp. 1-11.

[31]. Shinmura, S., (2015). Repeated Feature Selection Method for Microarray Data (5). Research Gate (5), Nov. 9, 2015, pp. $1-12$.

[32]. Shinmura, S., (2015). Comparison Fisher's LDF by JMP and Revised IP-OLDF by LINGO for Microarray Data (6). Research Gate (6), Nov. 11, 2015, pp. 1-10.

[33]. Shinmura, S., (2015). Matroska Trap of Feature Selection Method (7) -Golub et al. Microarray Data. Research Gate (7), Nov. 18, 2015, pp. 1-14.

[34]. Shinmura, S., (2015). Minimum Sets of Genes of Golub et al. Microarray Data (8). Research Gate (8), Nov. 22, 2015, pp. $1-12$.

[35]. Shinmura, S., (2015). Complete Lists of Small Matroska in Shipp et al. Microarray Data (9). Research Gate (9), Dec. 4, 2015, pp. 1-81.

[36]. Shinmura, S., (2015). Sixty-nine Small Matroska in Golub et al. Microarray Data (10). Research Gate, Dec. 4, pp. 1-58.

[37]. Shinmura, S., (2015). Simple Structure of Alon et al. et al. Microarray Data (11). Research Gate (11), Dec. 4, 2015 , pp. 1-34.

[38]. Shinmura, S., (2015). Feature Selection of Singh et al. Microarray Data (12). Research Gate (12), Dec. 6, 2015, pp. 1-89.

[39]. Shinmura, S., (2015). Final List of Small Matroska in Tian et al. Microarray Data. Research Gate (13), Dec. 7, pp. 1-160.

[40]. Shinmura, S., (2015). Final List of Small Matroska in Chiaretti et al. Microarray Data. Research Gate (14), Dec. 20, 2015, pp. 1-16.

[41]. Shinmura, S., (2015). "Matroska Feature Selection Methods for Microarray Data," Research Gate Free paper (15), pp.1-16.

[42]. Shinmura, S., (2015). "The Best Model of the Swiss Banknote Data-Validation by the 95\% CI of error rates and discriminant coefficients -," Optimization, and Information Computing, Vol.3, pp. 322-335. DOI: 10.19139/soic.20151202.

[43]. Shinmura, S., (2016). Matroska Feature Selection Method for Microarray Data. Biotechno 2016, 1-6, 2016.

[44]. Shinmura, S., (2016). The Basic Genes Sets (BGSs) for Microarray Data. Biotechno 2016, 1-6, 2016.

[45]. Shinmura, S., (2016). “The K-fold Cross Validation for Small Sample Method,” Data Analytic 2016, pp.1-6, 2016. (applied)

[46]. V.Vapnik, V., (1995). The Nature of Statistical Learning Theory. Springer-Verlag. 